Rheumatoid arthritis

\title{
Great expectations of modern RA treatment
}

\section{S Irvine, H C Capell}

\section{Rheumatological professionals and their patients should have high expectations of treatment}

$\mathrm{n}$ recent years the expectation of benefit of treatment of rheumatoid arthritis (RA) has evolved. Remission, as defined by the disease activity score (table 1), is now a goal for many clinicians. To achieve this it is standard practice to institute disease modifying antirheumatic drug (DMARD) treatment early in the disease course and to use combination DMARD treatment when single drugs prove ineffective. In addition, anti-tumour necrosis factor $\alpha$ $(\mathrm{TNF} \alpha)$ treatment has an established role in severe RA. However, despite these advances remission is achieved in only a minority of patients. Few randomised controlled trials (RCTs) in RA report remission data, which in itself is testament to the infrequency of remission. Why are expectations of greater benefit not realised?

In RCTs of the commonly used DMARDs methotrexate (MTX), sulfasalazine (SSZ), and leflunomide (LEF) as monotherapy, remission rates of $12.7-25 \%$ have been reported, depending on the population studied..$^{1-3}$ Although individual clinical responses to anti-TNF $\alpha$ monotherapy can be dramatic, remission rates in patients treated with anti-TNF $\alpha$ agents alone in RCTs have been disappointing, with $22.4 \%$ of patients with established disease achieving remission at 2 years. ${ }^{1}$

However, the combination of MTX and anti-TNF $\alpha$ treatment has provided more encouraging clinical results. At 2 years' follow up in the TEMPO study the combination of etanercept and MTX resulted in remission in $42.4 \%$ of patients with established RA compared with $25 \%$ remission with MTX or etanercept monotherapy. ${ }^{1}$ Similar rates of remission were also seen in the combination arm (MTX, SSZ, hydroxychloroquine (HCQ), and low dose prednisolone) of the FIN-RACo early RA study at 2 years. ${ }^{2}$

Although there are no published data on remission rates with triple treatment (MTX, SSZ, and HCQ) in established RA, two trials have demonstrated better clinical response with triple treatment than the combinations MTX/SSZ, MTX/
HCQ ${ }^{4}$ and SSZ/HCQ. ${ }^{5}$ In the last study of O'Dell et al an ACR70 response was seen in $26 \%$ of patients receiving triple treatment. ${ }^{4}$

It is well established that minimising disease activity reduces radiological progression and improves long term functional outcome. ${ }^{6}$ Acute phase reactants have been used as a marker for disease activity in studies and correlate with radiological progression. ${ }^{78}$ However, single measures of disease activity do not provide the full clinical picture. There is increasing evidence that low disease activity as defined by the composite measure of the Disease Activity Score (DAS) or 28 joint count DAS (DAS28; table 1) $)^{9-11}$ is associated with reduction in radiological progression..$^{12}$

\section{"Why are expectations of greater benefit not realised?"}

Of particular interest, Welsing et al investigated the longitudinal relationship between disease activity (measured by DAS and DAS28) and radiological progression in two independent follow up cohorts. ${ }^{13}$ They were able to describe different patterns of disease progression dependent on mean DAS, baseline Sharp score, and rheumatoid factor (RF) positivity. As is well established, positive RF and baseline joint damage were predictors of radiological progression. As expected, patients with constant high or fluctuating high DAS had significantly greater progression than those with constant low DAS. Of note, however, those with a fluctuating low DAS showed similar rates of progression to patients with a constant high DAS. These data not only support the approach of aiming for low disease activity but also call into question the "wait and see" approach of managing disease flares.

Traditionally RCTs of treatment in RA have been designed to compare specific pharmaceutical agents or combinations; the TICORA study however was an RCT designed to evaluate a strategy of targeting persistent disease activity with the aim of achieving tight control in early RA. ${ }^{14}$ The intensive treatment strategy was multifaceted. Patients had monthly assessments where systematic measurement of disease activity was made using the DAS, and DMARD treatment was escalated according to a protocol if the DAS was $>2.4$. Additionally intra-articular steroid was offered at each visit if there were swollen joints amenable to injection, and intramuscular steroid could be used as a "bridging treatment" within 3 months of starting a new DMARD. The control group received routine care in a standard rheumatology clinic.

When this strategy was used $67 \%$ of the group receiving intensive treatment achieved remission (DAS <1.6) at 18 months compared with $14 \%$ of the group receiving routine care. Additionally, the intensively treated group had less radiographic progression as measured by the total Sharp score and joint erosion score. Functional outcome measured by the Heath Assessment Questionnaire was also better in the intensive treatment arm.

Benefits of the intensive treatment strategy are likely to be multifactorial and which therapeutic intervention is the most important can be debated. However, setting a target of low disease activity, using regular systematic monitoring of disease activity, and adjusting DMARD treatment until that target is reached offer the best chance of achieving remission with the therapeutic agents currently available to us.

Translating these results into routine care is a problem. Is inertia in clinical practice or resistance from patients the obstacle to achieving targets?

\section{"Does inertia in clinical practice or patients' non-compliance prevent us from reaching targets?"}

In this month's Annals Fransen et al report a cluster randomised trial of systematic monitoring using the DAS28 compared with usual clinical care in RA. ${ }^{15}$ The study aimed at testing the hypothesis that use of systematic monitoring in the clinic setting would result in therapeutic changes leading to better disease control. In the DAS group physicians measured the DAS28 score at each visit and study guidelines stated that DMARD treatment should be increased (although specific changes in DMARDs were not per protocol) in patients with a DAS28 $>3.2$. The usual care (UC) group did not have DAS28 measurements and changes in treatment were at each rheumatologist's discretion. At the end of the 24 week study period the percentage of patients in the DAS group with low disease 
activity had increased from $13 \%$ at baseline to $31 \%$, whereas $16 \%$ of the UC group had low disease activity at 24 weeks compared with $12 \%$ at baseline. Although more patients in the DAS group had low disease activity at the study end point, there was no statistically significant difference in the mean changes in DAS between the groups.

The authors comment that the percentage of patients achieving low disease activity in the DAS group was less than expected and provide further interesting data about decision making during the study. Despite the study guidelines, DMARD changes in the DAS group were made by the participating rheumatologists at only $20 \%$ of visits where a DAS28 >3.2 was recorded. In the situations where DMARD treatment was not changed despite continuing disease activity the commonest reasons were physician based: (a) adopting a "wait and see" approach (84\%) and (b) disease activity was judged as sufficiently low by the rheumatologist despite the DAS28 score (54\%). This suggests that clinician inertia and low expectations contributed unfavourably to outcomes. In $31 \%$ of cases the patient did not wish to escalate treatment. Resolving this aspect requires patient education and encouragement. The TICORA protocol of frequent patient visits in the intensive arm was effective in this respect.

Some conclusions can be drawn from this study:

- Systematic monitoring in the clinic setting can improve disease control in individual patients

- Rheumatologists are reluctant to make decisions based on the DAS and prefer to use "clinical judgment"

- Some patients perceive increasing drug treatment to be less acceptable than continuing disease activity.

Why does systematic monitoring using the DAS or DAS28 result in improved disease control? Surely we are all aiming at achieving tight control of disease, and experienced rheumatologists do not need a "score" to aid decision making. However, each rheumatologist has his or her own perception of "low disease activity". The
DAS28 is a validated measure of disease activity with low interobserver variability. ${ }^{10}$ In routine rheumatology care, patients may not be seen on each occasion by the same physician. A documented objective measure of disease activity is more informative about the patient's disease course than subjective comments alone. When scoring global assessment of disease activity, physicians are most strongly influenced by acute phase reactants and swollen joints, giving less weight to tender joint counts and patient global assessment. ${ }^{16}$ Patients' impressions of disease activity have been shown to correlate well with the DAS $28^{17}$ and there is evidence from ultrasound studies that synovitis may be present in joints that do not have "clinical synovitis". ${ }^{18}$ Using the DAS28 may provide a more comprehensive assessment of disease activity.

\section{"Rheumatologists prefer to use their clinical judgment rather than make decisions based on the DAS"}

As demonstrated by Fransen et al the patient's role in decision making is also of importance. Leeb et al in a study of 207 patients with RA investigated patients' attitudes to the treatment. ${ }^{17}$ They found that patients wanted reduction of treatment at a mean DAS28 of 2.87 and did not want any change in treatment at a mean DAS28 of 3.52. It was not until a mean DAS28 of 4.92 was reached that increases in treatment were requested. The reasons for not wishing to change treatment are clearly complex. However, a possible explanation is that patients measure a good response to treatment by the amount of improvement in symptoms from baseline and may be satisfied with that alone. Patients may vary in their expectations of what can be achieved with treatment. Providing a target helps the patient to understand the rationale behind escalating drug treatment in the face of his or her own concerns about toxicity.

There are, however, some limitations to the use of the DAS or DAS28 in a routine clinical setting. Both scores were developed and validated to assess response in clinical trial cohorts ${ }^{9}{ }^{10}$ and in individual patients there are situa-

Table 1 Definitions of disease activity using DAS and DAS289-11

\begin{tabular}{lll}
\hline Definition & DAS & DAS 28 \\
\hline Remission & $<1.6$ & $<2.6$ \\
Low disease activity & $\leqslant 2.4$ & $\leqslant 3.2$ \\
Moderate disease activity & $>2.4$ and $\leqslant 3.7$ & $>3.2$ and $\leqslant 5.1$ \\
High disease activity & $>3.7$ & $>5.1$ \\
\hline
\end{tabular}

tions in which interpretation of the DAS is difficult. In patients with longstanding disease, eroded joints may be persistently tender in the absence of inflammation, and coexistent osteoarthritis may persistently raise the tender joint count. Patients with fibromyalgia are recognised to have persistently high DAS28 scores, and fibromyalgia is said to coexist in one in seven patients with RA. ${ }^{19}$ The DAS and DAS28 can be used in the majority of patients with RA as a reliable indicator of disease activity, but it is also important to record the separate components of the score and consider these in patients with DAS unresponsive to treatment. ${ }^{20}$

Adopting systematic monitoring in routine clinical practice has major resource implications. Although measuring and computing the DAS28 is not time consuming (estimated to take 5 minutes ${ }^{20}$ ) more treatment changes are made as a consequence. In the study of Fransen et al, DMARDs were adjusted twice as often in the DAS group as in the UC group. ${ }^{15}$ Similarly, patients in the intensive arm of the TICORA study were more likely to start new DMARDs and had more intra-articular injections than the group receiving routine care. Therefore the average duration of a single clinic consultation is increased. Additionally, patients in the TICORA intensive arm were seen monthly and it is likely that frequent assessment and intervention is important in achieving a state of constant low disease activity and the resultant slowing of radiological progression described by Welsing et al. ${ }^{13}$ Health economic assessment of the TICORA trial, however, showed that the increased outpatient costs of the intensive strategy were offset by increased community healthcare costs and inpatient costs in the routine care group and that the intervention was cost neutral. Nevertheless it can be difficult to convince health service managers that investing in resources early on saves disability and health costs at a later stage.

Increasing evidence shows that regular systematic monitoring of disease activity in RA, which aims at targeting persistent disease activity, can improve clinical outcomes and may slow radiological progression. Adopting this strategy in clinical practice requires significant changes in established rheumatology practice and increased rheumatology resources. It is important, however, that rheumatological professionals and their patients have high expectations of treatment, and that resources are sought to achieve targets of remission or low disease activity in the majority of patients with RA. 
Ann Rheum Dis 2005;64:1249-1251. doi: 10.1136/ard.2005.039339

......................

Authors' affiliations

S Irvine, H C Capell, Centre for Rheumatic Diseases, 84 Castle Street, Glasgow G4 OSF, UK

Correspondence to: Professor H C Capell, hilary.capell@northglasgow.scot.nhs.uk

\section{REFERENCES}

1 Klareskog L, van der Heijde D, de Jager JP, Gough A, Kalden J, Malaise M, et al. Therapeutic effect of the combination of etanercept and methotrexate compared with each treatment alone in patients with rheumatoid arthritis: double blind randomised trial. Lancet 2004:363:675-81.

2 Mottonen T, Hannonen P, Leirisalo-Repo M, Nissila $M$, Kautiainen $H$, Korpela $M$, et al. Comparison of combination therapy with single drug therapy in early rheumatoid arthritis: a randomised trial. Lancet 1999;353:1568-73.

3 Dougados M, Emery P, Lemmel E, de la Serna R, Zerbini CA, Brin S, et al. Efficacy and safety of leflunomide and predisposing factors for treatment response in patients with active rheumatoid arthritis: RELIEF 6-month data. J Rheumatol 2003;30:2572-9.

4 O'Dell JR, Leff R, Paulsen G, Haire C, Mallek J, Eckhoff PJ, et al. Treatment of rheumatoid arthritis with methotrexate and hydroxychloroquine, methotrexate and sulphasalazine or a combination of all three medications. Arthritis Rheum 2002;46:1164-70.
5 O'Dell J, Haire C, Erikson N, Drymalski W Palmer W, Eckhoff PJ, et al. Treatment of rheumatoid arthritis with methotrexate alone, sulfasalazine and hydroxychloroquine, or a combination of all three medications. NEngl J Med 1996;334: 1287-91

6 Fries JF, Williams CA, Morfeld D, Singh G Sibley J. Reduction of long-term disability in patients with rheumatoid arthritis by diseasemodifying antirheumatic drug-based treatment strategies. Arthritis Rheum 1996;39:616-22.

7 Van Leeuwen MA, van Rijswijk MH, van der Heijde DMFM, Te Meerman GJ, van Riel PL, Houtman PM, et al. The acute-phase response in relation to radiographic progression in early rheumatoid arthritis: a prospective study during the first three years of the disease. $\mathrm{Br} J$ Rheumato 1993;32(suppl 3):9-13.

8 Plant MJ, Williams AL, O'Sullivan MM, Lewis PA Coles EC, Jessop JD. Relationship between timeintegrated C-reactive protein levels and radiographic progression in patients with rheumatoid arthritis. Arthritis Rheum 2000:43:1473-7.

9 Van Gestel AM, Prevoo MLL, van 't Hof MA, van Rijswijk MH, van de Putte LB, van Riel PL. Development and validation of the European League Against Rheumatism response criteria for heumatoid arthritis. Arthritis Rheum 1996;39:34-40

10 Van Gestel AM, Haagsma CJ, van Riel PLCM. Validation of rheumatoid arthritis improvement criteria that include simplified joint counts. Arthritis Rheum 1998;41:1845-50.

11 Prevoo ML, van Gestel AM, van't Hof MA, van Rijswijk MH, van de Putte LB, van Riel PL. Remission in a prospective study of patients with rheumatoid arthritis: American Rheumatism Association preliminary remission criteria in relation to the disease activity score. Br J Rheumatol 1996;35:1101-5.
12 Svensson B, Shaufelberger C, Telemen A Theader J. Remission and response to early treatment of RA assessed by the Disease Activity Score. Rheumatology (Oxford) 2000;39:1031-6.

13 Welsing PMJ, Landewe RBM, van Riel PLCM, Boers $M$, van Gestel AM, van der Linden S, et al. The relationship between disease activity and radiologic progression in patients with rheumatoid arthritis - a longitudinal analysis. Arthritis Rheum 2004;50:2082-93.

14 Grigor C, Capell H, Stirling A, McMahon AD Lock $P, V a l l a n c e ~ R$, et al. Effect of a treatment strategy of tight control of rheumatoid arthritis (the TICORA study): a single-blind randomised controlled trial. Lancet 2004;364:263-9.

15 Fransen J, Bernelot Moens H, Speyer I, van Riel PLCM. Effectiveness of systematic monitoring of rheumatoid arthritis disease activity in daily practice: a multicentre, cluster randomised controlled trial. Ann Rheum Dis 2005;64:1294-8.

16 Nicolau G, Yogui MM, Vallochi TL, Gianini RJ, Laurindo IM, Novaes GS. Sources of discrepancy in patient and physican global assessments of rheumatoid arthritis disease activity. J Rheumatol 2004;31:1293-6.

17 Leeb BF, Ander I, Leder S, Leeb BA, Rintelen B. The patient's perspective and rheumatoid arthritis disease activity indexes. Rheumatology. 2005;44: 360-5; erratum, 2005;44:569.

18 Scheel AK, Hermann KG, Kahler E, Pasewaldt D Fritz J, Hamm B, et al. A novel ultrasonographic synovitis scoring system suitable for analyzing finger joint inflammation in rheumatoid arthritis. Arthritis Rheum 2005;52:733-43.

19 Leeb BF, Andel I, Sautner J, Nothnagl T, Rintelen B. The DAS28 in rheumatoid arthritis and fibromyalgia patients. Rheumatology (Oxford) 2004; 43:1504-7.

20 Uitz E, Franson J, Langenegger T, Stucki G. Clinical quality management in rheumatoid arthritis: putting theory into practice. Rheumatology (Oxford) 2000;39:542-9. 